\title{
Nonfattening condition for the generalized evolution by mean curvature and applications
}

\author{
SAMUEL BITON ${ }^{\dagger}$ \\ Laboratoire de Mathématiques et Physique Théorique - CNRS UMR 6083, Université de Tours, \\ Parc de Grandmont, 37200 Tours, France \\ PierRe CARDAliagueT ${ }^{*}$ \\ Département de Mathématiques, Faculté des Sciences, Université de Bretagne Occidentale, \\ 6, avenue Le Gorgeu, B.P. 809, 29285 Brest, France \\ OLIVIER LEY ${ }^{\S}$ \\ Laboratoire de Mathématiques et Physique Théorique - CNRS UMR 6083, Université de Tours, \\ Parc de Grandmont, 37200 Tours, France \\ [Received 14 September 2006 and in revised form 13 February 2007]

\begin{abstract}
We prove a nonfattening condition for a geometric evolution described by the level set approach. This condition is close to those of Soner [21] and Barles, Soner and Souganidis [5] but we apply it to some unbounded hypersurfaces. It allows us to prove uniqueness for the mean curvature equation for graphs with initial data convex at infinity, without any restriction on the growth at infinity, by seeing the evolution of the graph of a solution as a geometric motion.
\end{abstract}

\section{Introduction}

We consider the evolution $\Gamma_{t}$ of a given initial hypersurface $\Gamma_{0}$ of $\mathbb{R}^{N+1}$ moving according to the normal velocity

$$
\mathcal{V}_{(x, t)}=h\left(n_{x}, D n_{x}\right)
$$

where $n_{x}$ and $D n_{x}$ stand respectively for an oriented unit normal and the second fundamental form of $\Gamma_{t}$ at $x \in \Gamma_{t}$, and $h$ is the given evolution law. The hypothesis on $h$ will be introduced later but the key assumption in this paper is that $h$ is elliptic with respect to the second variable. Namely, if $X, Y$ are symmetric matrices, then

$$
X \leqslant Y \Rightarrow h\left(n_{x}, X\right) \geqslant h\left(n_{x}, Y\right)
$$

The most typical example we are interested in is the celebrated mean curvature evolution where

$$
\mathcal{V}_{(x, t)}=h\left(D n_{x}\right)=-\operatorname{Tr}\left(D n_{x}\right)
$$

\footnotetext{
†E-mail: biton@univ-tours.fr

‡E-mail: Pierre.Cardaliaguet@univ-brest.fr

$\S$ E-mail: ley@1mpt.univ-tours.fr
} 
To describe the evolution of $\Gamma_{t}$ according to (1), different ways have been proposed; see the book of Giga [13]. Here, we follow the level set approach introduced by Barles [1] and Osher and Sethian [20], developed first independently by Evans and Spruck [12] and Chen, Giga and Goto [8].

The level set approach has the advantage of being defined for all time $t \geqslant 0$, even past some singularities. We refer the reader to Section 2 for the definition and recall here that the evolution $\Gamma_{t}$ by the level set approach is given, at each time $t$, as the 0-level set of an auxiliary function, namely $\Gamma_{t}:=\left\{z \in \mathbb{R}^{N+1}: v(z, t)=0\right\}$, where $v: \mathbb{R}^{N+1} \times[0,+\infty) \rightarrow \mathbb{R}$ is the solution of a suitable parabolic partial differential equation of the form

$$
\frac{\partial v}{\partial t}+F\left(D v, D^{2} v\right)=0 \quad \text { in } \mathbb{R}^{N+1} \times(0,+\infty) .
$$

In this approach, one of the main issues is the so-called fattening phenomenon which happens when the front $\bigcup_{t \geqslant 0} \Gamma_{t} \times\{t\}$ has nonempty interior in $\mathbb{R}^{N+1} \times[0,+\infty)$. Some examples are known for which such a phenomenon arises (see Ilmanen [16] or Soner [21]). This fattening phenomenon is closely related to the nonuniqueness of the geometrical evolution (1). We refer to Ilmanen [16], Soner [21], Barles, Soner and Souganidis [5] or Barles, Biton and Ley [3] for further details.

Nevertheless, in [21] and [5] (see also [22] and [19]), the authors give sufficient conditions ensuring that the front never fattens. For instance, Soner proves that compact hypersurfaces which are strictly starshaped never fatten for evolutions with normal velocity of curvature type given (see Section 4.1).

Our aim in this article is to extend this method to unbounded sets which are entire graphs of functions from $\mathbb{R}^{N}$ into $\mathbb{R}$. Even if Soner's condition could be applied for some unbounded hypersurfaces (like convex graphs), it does not hold for the case we have in mind (graphs which are convex at infinity, see below). In [5], the authors give a more general condition for $C^{2}$ hypersurfaces but it is not clear how to extend it to unbounded cases.

To be more specific concerning our result, let $\Gamma_{0}$ be the boundary of an open subset $\Omega_{0}$ of $\mathbb{R}^{N+1}$ (notice that $\Gamma_{0}$ has empty interior). We prove that under suitable assumptions on the nonlinearity $F$ appearing in (4), the front never fattens if there exists a family $\left(\mathcal{A}_{\varepsilon}\right)_{\varepsilon>0}$ of affine dilations going to identity as $\varepsilon$ goes to 0 and such that

$$
\mathrm{d}\left(\Gamma_{0}, \mathcal{A}_{\varepsilon}\left(\Gamma_{0}\right)\right):=\inf \left\{|a-b|:(a, b) \in \Gamma_{0} \times \mathcal{A}_{\varepsilon}\left(\Gamma_{0}\right)\right\}>0 \quad \text { for any } \varepsilon>0 .
$$

This condition is close to the one of [5] but is stated in a more readable way which does not require the initial set $\Gamma_{0}$ to be $C^{2}$.

Our main contribution is to show that this condition can be used to prove the uniqueness of the evolution by mean curvature of entire graphs which are convex at infinity. We say that a continuous function $f: \mathbb{R}^{N} \rightarrow \mathbb{R}$ is convex at infinity if there exists $R>0$ such that, for any convex set $\mathcal{C} \subset \mathbb{R}^{N} \backslash B(0, R)$, the restriction $f: \mathcal{C} \rightarrow \mathbb{R}$ is convex. Our result is the following:

THEOREM 1.1 For any continuous initial data $u_{0}: \mathbb{R}^{N} \rightarrow \mathbb{R}$ which is convex at infinity (without any growth restriction), there exists a unique solution of the mean curvature equation for graphs

$$
\frac{\partial u}{\partial t}-\Delta u+\frac{\left\langle D^{2} u D u, D u\right\rangle}{1+|D u|^{2}}=0 \quad \text { in } \mathbb{R}^{N} \times(0,+\infty),
$$

with $u(\cdot, 0)=u_{0}$. 
The existence of a smooth solution $u \in C\left(\mathbb{R}^{N} \times[0,+\infty)\right) \cap C^{\infty}\left(\mathbb{R}^{N} \times(0,+\infty)\right)$ was proved by Ecker and Huisken [11]. It is very surprising that this result holds without any growth restriction at infinity.

The question of uniqueness of these solutions without a growth restriction at infinity is still open in the whole generality. Several partial results are known: In dimension $N=1$, the problem was completely solved independently by Chou and Kwong [9] and in [4]; in any dimension, uniqueness was proved in the following situations: with polynomial-type restrictions on the growth of $u_{0}$ in [2], when $u_{0}$ is radially symmetric in $\mathbb{R}^{N}$ in [7], and when $u_{0}$ is convex in $\mathbb{R}^{N}$ in [3]. After this paper was completed we learned that Ishii and Mikami had obtained in [18] a uniqueness result for the motion of a graph by $R$-curvature under some convexity at infinity type condition.

One could think that Theorem 1.1 is an easy generalization of the latter case but we point out that a very small perturbation of $u_{0}$ even on a compact set can modify the behaviour of the solution everywhere.

The uniqueness result of Theorem 1.1 holds in fact for more general quasilinear equations, the class of which is described in [3] (see Section 4.2). Moreover, we give an example (see Remark 4.1) of application of (5) to initial data which are not convex at infinity. It follows first that the set of functions convex at infinity is not the right class of uniqueness for equations like (6). Secondly, it emphasizes that our condition is of geometrical nature in the sense that we do not have any idea of how to prove such a result by pde methods.

The paper is organized as follows. In Section 2, we briefly recall the level-set approach. In Section 3 , we state and prove the sufficient condition (5). The last section is devoted to the proof of Theorem 1.1 and to its extension to other motions.

\section{Preliminaries about the level set approach}

In this section, we recall what we need about the level set approach and give the definition of the generalized evolution $\Gamma_{t}$. For more details see the book [13].

We start by introducing some definitions and notations. Given an open subset $\Omega_{0}^{+}$of $\mathbb{R}^{N+1}$, we say that $\left(\Gamma_{0}, \Omega_{0}^{+}, \Omega_{0}^{-}\right)$is an admissible partition if $\Gamma_{0}=\partial \Omega_{0}^{+}$( $\partial$ denotes the topological boundary) and $\Omega_{0}^{-}=\mathbb{R}^{N+1} \backslash\left(\Gamma_{0} \cup \Omega_{0}^{+}\right)$. Notice that $\Gamma_{0}$ has an empty interior. by

If $\left(\Gamma_{0}, \Omega_{0}^{+}, \Omega_{0}^{-}\right)$is an admissible partition, then the signed distance $d_{s}\left(\cdot, \Gamma_{0}\right)$ to $\Gamma_{0}$ is defined

$$
d_{s}\left(z, \Gamma_{0}\right):= \begin{cases}\mathrm{d}\left(z, \Gamma_{0}\right) & \text { if } z \in \Omega_{0}^{+}, \\ 0 & \text { if } z \in \Gamma_{0}, \\ -\mathrm{d}\left(z, \Gamma_{0}\right) & \text { if } z \in \Omega_{0}^{-},\end{cases}
$$

where $\mathrm{d}$ is the usual nonnegative distance in $\mathbb{R}^{N+1}$. Clearly $d_{S}\left(\cdot, \Gamma_{0}\right) \in U C\left(\mathbb{R}^{N+1}\right)$, where " $U C$ " denotes the uniformly continuous functions.

We aim at defining an evolution $\left(\Gamma_{t}, \Omega_{t}^{+}, \Omega_{t}^{-}\right)_{t} \geqslant 0$ starting from $\left(\Gamma_{0}, \Omega_{0}^{+}, \Omega_{0}^{-}\right)$where $\Gamma_{t}$ evolves with normal velocity 1 . Looking for an auxiliary function $v: \mathbb{R}^{N+1} \times[0,+\infty) \rightarrow \mathbb{R}$ satisfying, for every $t \geqslant 0$, the conditions

$$
\{v(\cdot, t)=0\}=\Gamma_{t}, \quad\{v(\cdot, t)>0\}=\Omega_{t}^{+} \quad \text { and } \quad\{v(\cdot, t)<0\}=\Omega_{t}^{-},
$$


we find that $v$ has to be a solution, at least formally, of the so-called level set equation for (1),

$$
\begin{cases}\frac{\partial v}{\partial t}+F\left(D v, D^{2} v\right)=0 & \text { in } \mathbb{R}^{N+1} \times(0,+\infty), \\ v(\cdot, 0)=v_{0} & \text { in } \mathbb{R}^{N+1},\end{cases}
$$

where, for instance, $v_{0}=d_{s}\left(\cdot, \Gamma_{0}\right)$, and

$$
F(p, X)=-|p| h\left(-\frac{p}{|p|},\left(-\frac{1}{|p|}\left(\mathrm{Id}-\frac{p \otimes p}{|p|^{2}}\right) X\right)_{\mid p^{\perp}}\right)
$$

for $p \in D(F) \subset \mathbb{R}^{N+1}$ and $X \in \mathcal{S}_{N+1}$. Here and below, $\mathcal{S}_{N+1}$ denotes the space of symmetric matrices of size $N+1$, and $M_{\mid p^{\perp}}$ is the restriction to the subspace $p^{\perp}$ of the linear map induced by $M \in \mathcal{S}_{N+1}$. Note that, in general, $F$ has singularities in the gradient variable and $D(F) \neq \mathbb{R}^{N+1}$.

From the very definition of $F$, it follows that

$$
F(\lambda p, \mu p \otimes p+\lambda M)=\lambda F(p, M) \quad \text { for all } p \in D(F), M \in \mathcal{S}_{N+1}, \lambda \geqslant 0, \mu \in \mathbb{R},
$$

and from (2), we have

$$
M \geqslant N \Rightarrow F(p, M) \leqslant F(p, N) \quad \text { for all } p \in D(F), M, N \in \mathcal{S}_{N+1} .
$$

It is worth noticing that $(9)$ implies that the equation in 77 is invariant under changes of function $v \mapsto \varphi \circ v$ with $\varphi^{\prime}>0$ whence we can work with bounded solutions of 7 .

Moreover (2)-(10) imply that (7) is degenerate elliptic and a maximum principle is expected. To avoid technicalities, we state the comparison principle as an assumption:

(H) If $v$ (respectively $w$ ) is a bounded uniformly continuous viscosity subsolution (respectively supersolution) of (7)-88) satisfying $v(\cdot, 0) \leqslant w(\cdot, 0)$, then $v \leqslant w$ in $\mathbb{R}^{N+1} \times[0,+\infty)$.

Assumptions on $F$ (or equivalently on $h$ ) which lead to this comparison principle are discussed in Section 4 We refer to [10] for a general discussion of the theory of viscosity solutions.

Now, we can state the following theorem and define the generalized evolution of $\Gamma_{0}$ with normal velocity given by (1):

Theorem 2.1 Suppose that 2 and $(\mathbf{H})$ hold. Then, for any $v_{0} \in U C\left(\mathbb{R}^{N+1}\right)$, there exists a unique $U C$ viscosity solution of (7). Moreover, set

$$
\left(\Gamma_{0}, \Omega_{0}^{+}, \Omega_{0}^{-}\right):=\left(\left\{v_{0}=0\right\},\left\{v_{0}>0\right\},\left\{v_{0}<0\right\}\right)
$$

and consider

$$
\left(\Gamma_{t}, \Omega_{t}^{+}, \Omega_{t}^{-}\right):=(\{v(\cdot, t)=0\},\{v(\cdot, t)>0\},\{v(\cdot, t)<0\}) .
$$

Then the family $\left(\Gamma_{t}, \Omega_{t}^{+}, \Omega_{t}^{-}\right)_{t} \geqslant 0$ is independent of the choice of $v_{0} \in U C\left(\mathbb{R}^{N+1}\right)$ satisfying 11 . Hence, it allows one to define $\left(\Gamma_{t}\right)_{t} \geqslant 0$ as the generalized evolution of $\Gamma_{0}$ with normal velocity (1) starting from the initial admissible partition $\left(\Gamma_{0}, \Omega_{0}^{+}, \Omega_{0}^{-}\right)$.

We do not prove the theorem here. Various assumptions on $F$ (or $h$ ) can be required in order that $(\mathbf{H})$ and the theorem holds true. In their celebrated papers, Evans and Spruck [12] and Chen, 
Giga and Goto [8] proved in particular Theorem 2.1 for the mean curvature evolution (3). In that case, the level set equation (7) is the well-known mean curvature equation

$$
\frac{\partial v}{\partial t}-\Delta v+\frac{\left\langle D^{2} v D v, D v\right\rangle}{|D v|^{2}}=0 \quad \text { in } \mathbb{R}^{N+1} \times(0,+\infty) .
$$

Other cases are treated in Giga, Goto, Ishii and Sato [14], Barles, Souganidis and Soner [5], Soner [21], Ishii and Souganidis [19], Ishii [17], Souganidis [23], Giga and Sato [15], and Barles, Biton and Ley [3]. See the book of Giga [13] and Section 4 for explicit examples.

\section{A sufficient condition for nonfattening}

In this section we give a condition on an initial hypersurface $\Gamma_{0}$ under which its generalized evolution never fattens. To this end we need the following assumption: there exists a positive continuous real-valued function $m$ such that $m(1)=1$ and

$$
F(p, \lambda M)=m(\lambda) F(p, M) \quad \text { for all } p \in D(F), M \in \mathcal{S}_{N+1}, \lambda>0,
$$

where $F$ is defined by 8 . Let $\mathcal{T}$ be the group of affine dilations of $\mathbb{R}^{N+1}$,

$$
\mathcal{T}=\left\{\mathcal{A}: \mathbb{R}^{N+1} \rightarrow \mathbb{R}^{N+1}: \mathcal{A}(z)=\lambda z+z_{0}, \lambda \in \mathbb{R} \backslash\{0\}, z_{0} \in \mathbb{R}^{N+1}\right\} .
$$

Lemma 3.1 Assume that $(\mathbf{H})$ and $(12)$ hold. Suppose that $D(F)$ is invariant under dilations, $\left(\Gamma_{0}, \Omega_{0}^{+}, \Omega_{0}^{-}\right)$is an admissible initial partition, and $\mathcal{A} \in \mathcal{T}$ with coefficient $\lambda \neq 0$. Let $v$ (respectively $v_{\mathcal{A}}$ ) be the solution of (7) associated to $d_{s}\left(\cdot, \Gamma_{0}\right)$ (respectively $d_{s}\left(\cdot, \mathcal{A}\left(\Gamma_{0}\right)\right.$ )). Then

$$
v_{\mathcal{A}}(z, t)=\lambda v\left(\mathcal{A}^{-1} z, \frac{t}{\lambda m(\lambda)}\right) .
$$

Proof. Suppose that $\mathcal{A} z=\lambda z+z_{0}$. Let $v_{0}=d_{s}\left(\cdot, \Gamma_{0}\right)$ (respectively $w_{0}=d_{s}\left(\cdot, \mathcal{A}\left(\Gamma_{0}\right)\right)$ ) and $v$ (respectively $w$ ) be the unique uniformly continuous solution of (7) with initial data $v_{0}$ (respectively $\left.w_{0}\right)$. Observing that $w_{0}=\lambda v_{0} \circ \mathcal{A}^{-1}$ we set

$$
w(z, t)=\lambda v\left(\frac{z-z_{0}}{\lambda}, \frac{t}{\lambda m(\lambda)}\right) .
$$

Using (9) and (12), one checks that $w$ is a solution of (7) with initial data $w_{0}$. Therefore we get the lemma by the uniqueness result for 77 .

Let $A, B$ be two subsets of $\mathbb{R}^{N+1}$. We define the minimum distance $\mathrm{d}(A, B)$ between $A$ and $B$ by

$$
\mathrm{d}(A, B):=\inf _{a \in A, b \in B}|a-b| .
$$

LEMma 3.2 For any admissible partitions $\left(\Gamma_{0}, \Omega_{0}^{+}, \Omega_{0}^{-}\right)$and $\left(\tilde{\Gamma}_{0}, \tilde{\Omega}_{0}^{+}, \tilde{\Omega}_{0}^{-}\right)$, if $\tilde{\Omega}_{0}^{+} \cup \tilde{\Gamma}_{0} \subset \Omega_{0}^{+}$ then

$$
\mathrm{d}\left(\Gamma_{0}, \tilde{\Gamma}_{0}\right) \geqslant \eta \geqslant 0 \Rightarrow d_{s}\left(\cdot, \Gamma_{0}\right) \geqslant d_{s}\left(\cdot, \tilde{\Gamma}_{0}\right)+\eta .
$$




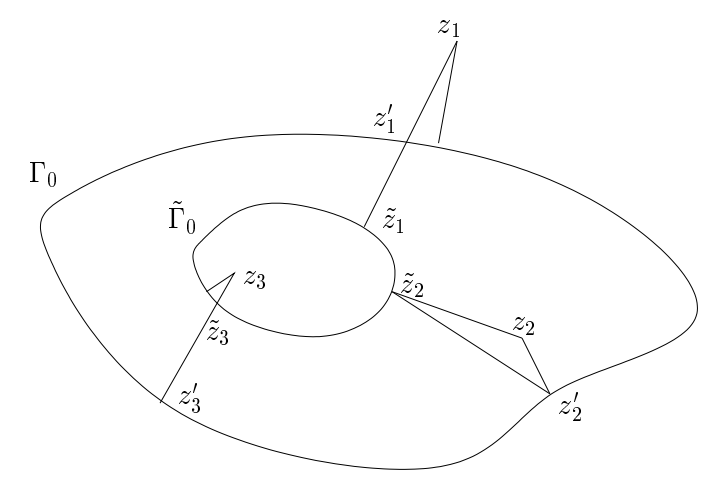

FIG. 1. The different cases under consideration in the proof of Lemma 3.2

REMARK 3.1 A consequence of the comparison assumption $(\mathbf{H})$ and Lemma 3.2 is an inclusion principle which roughly states that, if $\Omega_{0}^{+}$and $\tilde{\Omega}_{0}^{+}$are such that $\Omega_{0}^{+} \subset \tilde{\Omega}_{0}^{+}$, then this inclusion remains true for all time: $\Omega_{t}^{+} \subset \tilde{\Omega}_{t}^{+}$. We point out that this inclusion principle is an important underlying property of geometrical evolutions satisfying (2). For a general study of geometrical evolutions which satisfy this principle, see Barles and Souganidis [6].

Proof of Lemma 3.2 We distinguish several cases according to the position of $z$ (see Figure 11).

CASE 1: $z_{1} \in \Omega_{0}^{-} \cup \Gamma_{0} . \quad$ Let $a=\mathrm{d}\left(z_{1}, \Gamma_{0}\right)$ and $b=\mathrm{d}\left(z_{1}, \tilde{\Gamma}_{0}\right)=\left|z_{1}-\tilde{z}_{1}\right|$, with $\tilde{z}_{1} \in \tilde{\Gamma}_{0}$. Consider a point $z_{1}^{\prime} \in\left[z_{1}, \tilde{z}_{1}\right] \cap \Gamma_{0}$. We have

$$
d_{s}\left(z_{1}, \Gamma_{0}\right)-d_{s}\left(z_{1}, \tilde{\Gamma}_{0}\right)=-a+b=-a+\left|z_{1}-z_{1}^{\prime}\right|+\left|z_{1}^{\prime}-\tilde{z}_{1}\right| .
$$

But $\left|z_{1}-z_{1}^{\prime}\right| \geqslant \mathrm{d}\left(z_{1}, \Gamma_{0}\right)=a$ and $\left|z_{1}^{\prime}-\tilde{z}_{1}\right| \geqslant d_{e}\left(\Gamma_{0}, \tilde{\Gamma}_{0}\right) \geqslant \eta$; therefore $d_{s}\left(z_{1}, \Gamma_{0}\right)-d_{s}\left(z_{1}, \tilde{\Gamma}_{0}\right) \geqslant \eta$. CASE 2: $z_{2} \in \Omega_{0}^{+} \cap \tilde{\Omega}_{0}^{-} . \quad$ Let $a=\mathrm{d}\left(z_{2}, \Gamma_{0}\right)=\left|z_{2}-z_{2}^{\prime}\right|$ with $z_{2}^{\prime} \in \Gamma_{0}$, and $b=\mathrm{d}\left(z_{2}, \tilde{\Gamma}_{0}\right)=\left|z_{2}-\tilde{z}_{2}\right|$ with $\tilde{z}_{2} \in \tilde{\Gamma}_{0}$. We have

$$
d_{s}\left(z_{2}, \Gamma_{0}\right)-d_{s}\left(z_{2}, \tilde{\Gamma}_{0}\right)=a+b \geqslant\left|z_{2}^{\prime}-\tilde{z}_{2}\right| \geqslant \eta .
$$

CASE 3: $z_{3} \in \tilde{\Omega}_{0}^{-} \cup \tilde{\Gamma}_{0}$. Let $a=\mathrm{d}\left(z_{3}, \Gamma_{0}\right)=\left|z_{3}-z_{3}^{\prime}\right|$ with $z_{3}^{\prime} \in \Gamma_{0}$, and $b=\mathrm{d}\left(z_{3}, \tilde{\Gamma}_{0}\right)$. Consider a point $\tilde{z}_{3} \in\left[z_{3}, z_{3}^{\prime}\right] \cap \tilde{\Gamma}_{0}$. We have

$$
d_{s}\left(z_{3}, \Gamma_{0}\right)-d_{s}\left(z_{3}, \tilde{\Gamma}_{0}\right)=a-b=\left|z_{3}-\tilde{z}_{3}\right|+\left|\tilde{z}_{3}-z_{3}^{\prime}\right|-b .
$$

But $\left|\tilde{z}_{3}-z_{3}^{\prime}\right| \geqslant d_{e}\left(\Gamma_{0}, \tilde{\Gamma}_{0}\right) \geqslant \eta$ and $\left|z_{3}-\tilde{z}_{3}\right| \geqslant \mathrm{d}\left(z_{3}, \tilde{\Gamma}_{0}\right)=b$; thus $d_{s}\left(z_{3}, \Gamma_{0}\right)-d_{s}\left(z_{3}, \tilde{\Gamma}_{0}\right) \geqslant \eta$, which completes the proof of Lemma 3.2

Now, we can state the main result of this section.

THEOREM 3.1 Let $\left(\Gamma_{0}, \Omega_{0}^{+}, \Omega_{0}^{-}\right)$be an admissible initial partition and assume that Theorem 2.1 and (12) hold. If there exists a family $\left(\mathcal{A}_{\varepsilon}\right)_{\varepsilon>0} \subset \mathcal{T}$ and a sequence $\left(\eta_{\varepsilon}\right)_{\varepsilon>0}$ of positive numbers such that

$$
\mathcal{A}_{\varepsilon} \underset{\varepsilon \rightarrow 0}{\longrightarrow} \operatorname{Id} \quad \text { and } \quad \mathrm{d}\left(\Gamma_{0}, \mathcal{A}_{\varepsilon}\left(\Gamma_{0}\right)\right) \geqslant \eta_{\varepsilon}>0 \quad \text { for } \varepsilon>0,
$$

then the front $\bigcup_{t \geqslant 0} \Gamma_{t} \times\{t\}$ has empty interior in $\mathbb{R}^{N+1} \times[0,+\infty)$. 
Proof. Let $v$ and $v_{\mathcal{A}_{\varepsilon}}$ be the uniformly continuous viscosity solutions of (7) associated to the initial data $d_{s}\left(\cdot, \Gamma_{0}\right)$ and $d_{s}\left(\cdot, \mathcal{A}_{\varepsilon}\left(\Gamma_{0}\right)\right)$. From Lemma 3.1 , for every $(z, t) \in \mathbb{R}^{N+} \times[0,+\infty)$, we have

$$
v_{\mathcal{A}_{\varepsilon}}(z, t)=\lambda_{\varepsilon} v\left(\mathcal{A}_{\varepsilon}^{-1} z, \frac{t}{\lambda_{\varepsilon} m\left(\lambda_{\varepsilon}\right)}\right),
$$

where $\lambda_{\varepsilon}$ is the coefficient of $\mathcal{A}_{\varepsilon}$. Next, from (13), Lemma 3.2 and the comparison principle (H), we get, for any $\varepsilon>0$,

$$
v(z, t) \geqslant v_{\mathcal{A}_{\varepsilon}}(z, t)+\eta_{\varepsilon} .
$$

Therefore

$$
v(z, t) \geqslant \lambda_{\varepsilon} v\left(\mathcal{A}_{\varepsilon}^{-1} z, \frac{t}{\lambda_{\varepsilon} m\left(\lambda_{\varepsilon}\right)}\right)+\eta_{\varepsilon}
$$

Assume now that the front $\bigcup_{t} \geqslant 0 \Gamma_{t} \times\{t\}$ has nonempty interior in $\mathbb{R}^{N+1} \times[0,+\infty)$. It follows that there is some $\left(z_{0}, t_{0}\right) \in \mathbb{R}^{N+1} \times(0,+\infty)$ and some $r>0$ such that

$$
v \equiv 0 \quad \text { in } B\left(z_{0}, r\right) \times\left[t_{0}-r, t_{0}+r\right] .
$$

Since $\mathcal{A}_{\varepsilon} \rightarrow \mathrm{Id}$, one has $\lambda_{\varepsilon} \rightarrow 1$ and then, for $\varepsilon$ sufficiently small,

$$
\left(\mathcal{A}_{\varepsilon}^{-1} z_{0}, \frac{t_{0}}{\lambda_{\varepsilon} m\left(\lambda_{\varepsilon}\right)}\right) \in B\left(\left(z_{0}, t_{0}\right), r\right) \times\left[t_{0}-r, t_{0}+r\right] .
$$

Writing (14) at the point $\left(z_{0}, t_{0}\right)$, we obtain a contradiction which ends the proof.

\section{Application to uniqueness results}

In this section we give some applications of Theorem 3.1. The first application is known and concerns the evolution of compact sets. The second, which is the main result and the motivation of this work, gives new uniqueness results for quasilinear parabolic pdes.

We recall some explicit assumptions on the evolution law $h$ which appears in (1) or, equivalently, on $F$ defined by (8) which imply the comparison assumption $(\mathbf{H})$.

\subsection{Uniqueness of generalized evolutions of compact sets}

We show that Theorem 3.1 applies to recover some results of [21] and [19]. We suppose first

(H1) The evolution law $h$ is linear with respect to the second fundamental form, i.e. $h=$ $-\operatorname{Tr}\left(G\left(n_{x}\right) D n_{x}\right)$, and $G: S^{N} \rightarrow \mathcal{S}_{N+1}^{+}$is continuous, where $S^{N}=\left\{\xi \in \mathbb{R}^{N+1}:|\xi|=1\right\}$ is the unit sphere and $\mathcal{S}_{N+1}^{+}$is the set of nonnegative symmetric matrices of size $N+1$.

LEMMA 4.1 ([21]) Under assumption (H1), (H) holds.

Noticing that 12 holds with $m(r)=r$, we have

THEOREM 4.1 ([21]) Let $\left(\Gamma_{0}, \Omega_{0}^{+}, \Omega_{0}^{-}\right)$be an admissible partition such that $\Gamma_{0}$ has empty interior and evolves with velocity $\sqrt{1}$ ) satisfying $(\mathbf{H 1})$. Suppose that $\overline{\Omega_{0}^{+}}$is a compact subset which is strictly starshaped, i.e. there exists $z_{0} \in \Omega_{0}^{+}$such that, for all $z \in \overline{\Omega_{0}^{+}},\left[z_{0}, z\left[\subset \Omega_{0}^{+}\right.\right.$. Then $\bigcup_{t \geqslant 0} \Gamma_{t} \times\{t\}$ has empty interior. 
This theorem was proved by Soner [21, Theorem 9.3]. Our proof is basically the same so we only sketch it. Up to a translation, we can suppose that $z_{0}=0$ and we check that the family $\mathcal{A}_{\varepsilon}(z)=(1-\varepsilon) z$ for $\varepsilon \in(0,1)$ satisfies $(13)$. We conclude by Theorem 3.1. It is worth pointing out again that the previous result includes the mean curvature motion (3).

We present another example of motion, namely the motion by Gaussian curvature:

(H2) The evolution law $h$ is given by $h\left(D n_{x}\right)=\kappa_{1}^{+} \ldots \kappa_{N}^{+}$where $\kappa_{1}, \ldots, \kappa_{N}$ are the principal curvatures of $\Gamma_{t}$ (the eigenvalues of $D n_{x}$ ) and $r^{+}:=\max \{r, 0\}$.

In this case, the level set equation (7)-(8) reads

$$
\frac{\partial v}{\partial t}-|D v| \operatorname{det}_{+}\left[\frac{1}{|D v|}\left(\operatorname{Id}-\frac{D v \otimes D v}{|D v|^{2}}\right) D^{2} v\left(\operatorname{Id}-\frac{D v \otimes D v}{|D v|^{2}}\right)+\frac{D v \otimes D v}{|D v|^{2}}\right]=0
$$

in $\mathbb{R}^{N+1} \times(0,+\infty)$, where, for any symmetric matrix $X \in \mathcal{S}_{N+1}$ with eigenvalues $\lambda_{1}, \ldots, \lambda_{N+1}$, $\operatorname{det}_{+}(X)=\lambda_{1}^{+} \cdots \lambda_{N+1}^{+}$. Under assumption (H2), (H) holds and therefore Theorem 2.1 applies (see [19]). Moreover (12) holds with $m(r)=r^{N}$. Thus Theorem 4.1 holds true with the same proof. In this way, we recover [19], Proposition 3.4] without assuming $C^{2}$ regularity for $\Gamma_{0}$.

\subsection{Uniqueness of solutions of quasilinear parabolic pdes}

We turn to our main application. Consider the following pde:

$$
\left\{\begin{array}{l}
\frac{\partial u}{\partial t}-\operatorname{Tr}\left[b(D u) D^{2} u\right]=0 \quad \text { in } \mathbb{R}^{N} \times(0,+\infty) \\
u(\cdot, 0)=u_{0} \in C\left(\mathbb{R}^{N}\right)
\end{array}\right.
$$

where $b: \mathbb{R}^{N} \rightarrow \mathcal{S}_{N}^{+}$and $\mathcal{S}_{N}^{+}$is the set of nonnegative symmetric matrices of size $N$. Note that equation (15) is quasilinear parabolic (possibly degenerate). Existence of solutions to [15] is not the point here; we refer to [11], [9], [3] for quite general results for any continuous initial data $u_{0}$ without any growth restriction at infinity. The question we address here is the uniqueness of these solutions.

In [3] we show that under suitable assumptions on the diffusion matrix $b$ (see below), the graphs of the solutions of 15 are hypersurfaces of $\mathbb{R}^{N+1}$ moving according to a geometrical law of type (1). This makes it possible to define the generalized evolution $\Gamma_{t}$ of

$$
\Gamma_{0}=\operatorname{Graph}\left(u_{0}\right)=\left\{\left(x, u_{0}(x)\right): x \in \mathbb{R}^{N}\right\} \subset \mathbb{R}^{N+1},
$$

and we prove that the graphs of all the continuous viscosity solutions of $(15)$ are contained in the front $\bigcup_{t \geqslant 0} \Gamma_{t} \times\{t\}$. It follows that the uniqueness of continuous viscosity solutions is equivalent to the nonfattening of the front (see [3, Theorem 6.2]).

In this case, the level set equation is (7) with

$$
F\left(D v, D^{2} v\right)=-\operatorname{Tr}\left[b\left(-\frac{D_{x} v}{D_{y} v}\right)\left(D_{x x}^{2} v-2 D_{x y}^{2} \otimes \frac{D_{x} v}{D_{y} v}+D_{y y} v \frac{D_{x} v}{D_{y} v} \otimes \frac{D_{x} v}{D_{y} v}\right)\right],
$$

and $D(F)=\left\{p=\left(p_{1}, \ldots, p_{N+1}\right) \in \mathbb{R}^{N+1}: p_{N+1}=0\right\}$. The precise assumptions we need are 
(H3) The map $b: \mathbb{R}^{N} \rightarrow \mathcal{S}_{N}^{+}$is continuous, there exists a constant $C>0$ such that $|b(q)|+$ $|b(q) q|+|\langle b(q) q, q\rangle| \leqslant C$ for all $q \in \mathbb{R}^{N}$, and there exists a continuous map $b_{\infty}:\left\{\xi \in \mathbb{R}^{N}\right.$ : $|\xi|=1\} \rightarrow \mathcal{S}_{N}^{+}$such that $b_{\infty}(q)=\lim _{\lambda \rightarrow \pm \infty} b(\lambda q)$.

Lemma 4.2 ([3]) Under assumption (H3), (H) holds.

Roughly speaking, these assumptions allow us to control the singularities of $F$ in order to prove the comparison result and apply Theorem 2.1 Our main result is the following:

THEOREM 4.2 Assume that $F$ defined by 16 satisfies $(\mathbf{H 3})$. If $u_{0} \in C\left(\mathbb{R}^{N}\right)$ is convex at infinity, then 15 has at most one continuous viscosity solution.

Before giving the proof of the theorem, we make some comments. The typical example we have in mind is

$$
b(p)=\mathrm{Id}-\frac{p \otimes p}{1+|p|^{2}} .
$$

In this case, (15) reduces to the mean curvature equation for graphs (6) and therefore the above theorem includes as a particular case Theorem 1.1 which is the motivation of this work. For other examples, see [3]. A similar result for motion of a graph by $R$-curvature with initial data convex at infinity was obtained by Ishii and Mikami [18].

REMARK 4.1 (A function $u_{0}$ which satisfies the assumptions of Theorem 3.1 but is not convex at infinity) Let $f, g \in C\left(\mathbb{R}^{N}\right)$ be such that $f$ is convex in $\mathbb{R}^{N}$ and $g(x) \rightarrow 0$ as $|x| \rightarrow+\infty$. Set $u_{0}=\max \{f(x), g(x)\}$. Then $u_{0} \in C\left(\mathbb{R}^{N}\right)$ is not necessarily convex at infinity (for $N=1$, take for instance $f(x)=e^{x^{3}}$ and $\left.g(x)=(\sin x) / x\right)$. But $u_{0}$ satisfies 13 . We do not give the proof since it is close to the proof of Theorem 4.2 This example shows that Theorem 3.1 applies to a larger class of graphs than those convex at infinity.

Proof of Theorem 4.2. From [3, Theorem 6.2], it is sufficient to prove that the generalized evolution $\Gamma_{t}$ of $\Gamma_{0}:=\mathrm{Graph}\left(u_{0}\right)$ does not develop an interior. We proceed in two steps.

STEP 1. To emphasize the main ideas without technicalities, we first suppose that $u_{0}$ is convex in $\mathbb{R}^{N}$. Up to translating $\Gamma_{0}$, we can assume that there exists $\rho>0$ such that $\overline{B(0, \rho)} \subset \operatorname{Epi}\left(u_{0}\right)=$ $\left\{(x, r) \in \mathbb{R}^{N+1}: r \geqslant u_{0}(x)\right\}$. Consider the family $\left(\mathcal{A}_{\varepsilon}\right)_{\varepsilon>0} \subset \mathcal{T}$ defined by

$$
\mathcal{A}_{\varepsilon}=(1+\varepsilon) \operatorname{Id}, \quad 0<\varepsilon<1,
$$

and set $\Gamma_{0}^{\varepsilon}=\mathcal{A}_{\varepsilon}\left(\Gamma_{0}\right)$.

We aim at applying Theorem 3.1. We claim there exists a sequence $\left(\eta_{\varepsilon}\right)_{\varepsilon>0}$ of positive numbers such that

$$
\mathrm{d}\left(\Gamma_{0}, \mathcal{A}_{\varepsilon}\left(\Gamma_{0}\right)\right) \geqslant \eta_{\varepsilon} .
$$

Indeed, let $z_{0}=\left(x_{0}, y_{0}\right) \in \Gamma_{0}$ and $z_{\varepsilon}=\mathcal{A}_{\varepsilon}\left(z_{0}\right)=(1+\varepsilon) z_{0}$. For $\xi$ in the convex subdifferential $\partial u_{0}\left(x_{0}\right)$ of $u_{0}$ at $x_{0}$, consider the support hyperplane

$$
H_{0}=\left\{z=(x, y) \in \mathbb{R}^{N+1}: y=\left\langle\xi, x-x_{0}\right\rangle+y_{0}\right\}
$$

to Epi $\left(u_{0}\right)$ passing through $z_{0}$ (see Figure 2). Since $u_{0}$ is convex, Epi $\left(u_{0}\right)$ lies in the half-space $\left\{y \geqslant\left\langle\xi, x-x_{0}\right\rangle+y_{0}\right\}$; it follows that

$$
\mathrm{d}\left(z_{\varepsilon}, \Gamma_{0}\right) \geqslant \mathrm{d}\left(z_{\varepsilon}, H_{0}\right)=\mathrm{d}\left(H_{\varepsilon}, H_{0}\right),
$$




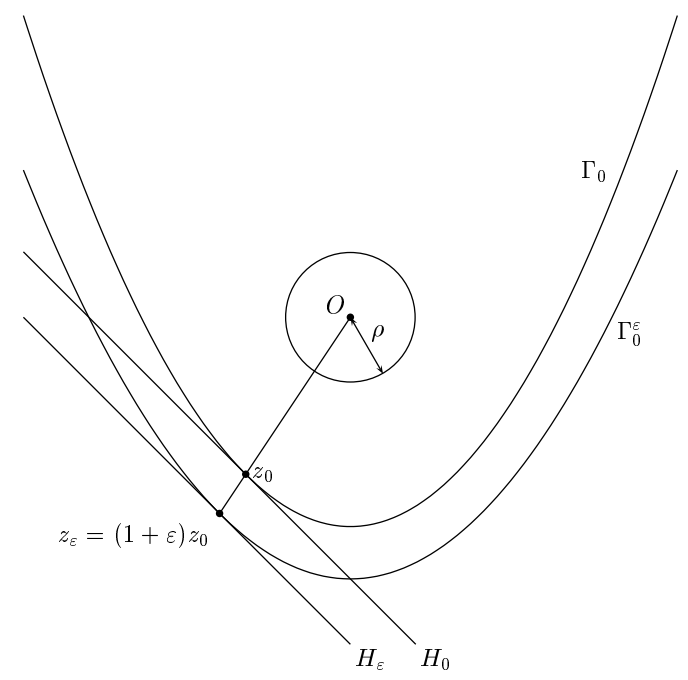

FIG. 2. $\Gamma_{0}=\operatorname{Graph}\left(u_{0}\right)$ with $u_{0}$ convex.

where $H_{\varepsilon}=\left\{z=(x, y): y=\left\langle\xi, x-(1+\varepsilon) x_{0}\right\rangle+(1+\varepsilon) y_{0}\right\}$ is the hyperplane parallel to $H_{0}$ and passing through $z_{\varepsilon}$. Noticing that $H_{\varepsilon}=\mathcal{A}_{\varepsilon}\left(H_{0}\right)$, we obtain

$$
\mathrm{d}\left(H_{\varepsilon}, H_{0}\right) \geqslant \varepsilon \mathrm{d}\left(0, H_{0}\right) \geqslant \rho \varepsilon .
$$

Finally, for every $z_{\varepsilon} \in \Gamma_{0}^{\varepsilon}, \mathrm{d}\left(z_{\varepsilon}, \Gamma_{0}\right) \geqslant \rho \varepsilon$; therefore $(17)$ holds with $\eta_{\varepsilon}=\rho \varepsilon$. It follows that assumption $(13)$ of Theorem 3.1 holds and we obtain the desired conclusion.

Note that Step 1 provides a new proof of [3, Theorem 10.1].

STEP 2: The general case. From now on, we assume that the map $u_{0}$ is convex at infinity. Let $R_{0}>0$ be some constant such that $u_{0}$ is convex on any convex subset of $\mathbb{R}^{N} \backslash B\left(0, R_{0}\right)$. For $x \in \mathbb{R}^{N}$ with $|x|>R_{0}$, we define the subdifferential $\partial u_{0}(x)$ as the subdifferential at $x$ of the restriction of $u_{0}$ to any convex neighbourhood of $x$ contained in $\mathbb{R}^{N} \backslash B\left(0, R_{0}\right)$. Since the notion of subdifferential is local, $\partial u_{0}(x)$ is well defined. Let us point out that $\partial u_{0}(x)$ enjoys the following property: if $p \in$ $\partial u_{0}(x)$, then

$$
\forall y \in \mathbb{R}^{N} \text { with }[x, y] \cap \overline{B\left(0, R_{0}\right)}=\emptyset, \quad u_{0}(y) \geqslant u_{0}(x)+\langle p, y-x\rangle .
$$

Moreover $\partial u_{0}(x)$ is nonempty as soon as $|x|>R_{0}$.

With this in mind, let us state a preliminary (and technical) lemma (for the proof and a comment, see below).

Lemma 4.3 Assume that $u_{0}$ is convex at infinity. Then there is some radius $R>0$ and some constant $c \in \mathbb{R}$ such that

(i) $u_{0}$ is convex on any convex subset of $\mathbb{R}^{N} \backslash B(0, R)$,

(ii) for any $x \notin B(0, R+1)$ and any $p \in \partial u_{0}(x)$,

$$
u_{0}(x)-\langle p, x\rangle \leqslant-|p|+c .
$$


For any $\varepsilon>0$ and any $\lambda \in(0,1)$, set

$$
u_{\varepsilon, \lambda}(x)=(1-\lambda)\left[u_{0}\left(\frac{x}{1-\lambda}\right)+\varepsilon\right] .
$$

The graph of $u_{\varepsilon, \lambda}$ is the image of the graph of $u_{0}$ under the similitude $\mathcal{A}_{\varepsilon, \lambda}$ defined by

$$
\mathcal{A}_{\varepsilon, \lambda}(z)=(1-\lambda)(z+(0, \varepsilon)) .
$$

Note that $\mathcal{A}_{\varepsilon, \lambda} \rightarrow$ Id as $\varepsilon, \lambda \rightarrow 0$.

To conclude applying Theorem 3.1, it is sufficient to prove the following claim: for any $\varepsilon>0$, there is some $\lambda_{\varepsilon} \in(0,1)$ such that, for any $\lambda \in\left(0, \lambda_{\varepsilon}\right)$,

$$
\mathrm{d}\left(\operatorname{Graph}\left(u_{0}\right), \operatorname{Graph}\left(u_{\varepsilon, \lambda}\right)\right)>0 .
$$

Let $R$ and $c$ be as in Lemma 4.3 . Without loss of generality, up to some translation, we can assume that $c=-1$. Thus we have

$$
\forall x \notin B(0, R+1), \forall p \in \partial u_{0}(x), \quad u_{0}(x)-\langle p, x\rangle \leqslant-(1+|p|) .
$$

Since $u_{0}$ is continuous, we have

$$
\forall z \in \mathbb{R}^{N}, \quad \mathrm{~d}\left(\left(z, u_{0}(z)\right), \operatorname{Graph}\left(u_{0}\right)+(0, \varepsilon)\right)>0 .
$$

Therefore, $\gamma_{\varepsilon}=\min _{|z| \leqslant R+1} \mathrm{~d}\left(\left(z, u_{0}(z)\right)\right.$, $\left.\operatorname{Graph}\left(u_{0}\right)+(0, \varepsilon)\right)$ is positive.

Let $x, y \in \mathbb{R}^{N}$. We want to estimate from below $\left|\left(x, u_{0}(x)\right)-\left(y, u_{\varepsilon, \lambda}(y)\right)\right|$ by some constant independent of $x$ and $y$. For this, let us first assume that $x \in B(0, R+1)$. We can also suppose that $|x-y| \leqslant 1$. Then

$$
\left|\left(x, u_{0}(x)\right)-\left(y, u_{\varepsilon, \lambda}(y)\right)\right| \geqslant\left|\left(x, u_{0}(x)\right)-\left(y, u_{0}(y)+\varepsilon\right)\right|-\left|\left(u_{0}(y)+\varepsilon\right)-(1-\lambda)\left(u_{0}\left(\frac{y}{1-\lambda}\right)+\varepsilon\right)\right| .
$$

Since $\left(y, u_{0}(y)+\varepsilon\right)$ belongs to $\operatorname{Graph}\left(u_{0}\right)+(0, \varepsilon)$, we have

$$
\left|\left(x, u_{0}(x)\right)-\left(y, u_{\varepsilon, \lambda}(y)\right)\right| \geqslant \gamma_{\varepsilon}-\left[\lambda \varepsilon+\lambda\left|u_{0}\left(\frac{y}{1-\lambda}\right)\right|+\left|u_{0}(y)-u_{0}\left(\frac{y}{1-\lambda}\right)\right|\right] .
$$

We can choose $\lambda_{\varepsilon}>0$ small enough such that, for every $\lambda \in\left(0, \lambda_{\varepsilon}\right)$,

$$
\forall y \in B(0, R+2), \quad \lambda \varepsilon+\lambda\left|u_{0}\left(\frac{y}{1-\lambda}\right)\right|+\left|u_{0}(y)-u_{0}\left(\frac{y}{1-\lambda}\right)\right| \leqslant \gamma_{\varepsilon} / 2 .
$$

This leads to

$$
\left|\left(x, u_{0}(x)\right)-\left(y, u_{\varepsilon, \lambda}(y)\right)\right| \geqslant \gamma_{\varepsilon} / 2 .
$$

Let us now assume that $x \notin B(0, R+1)$. Let us choose some $p \in \partial u_{0}(x)$. Since $|y-x| \leqslant 1$ and $|x| \geqslant R+1$, the segment $[x, y /(1-\lambda)]$ is a subset of $\mathbb{R}^{N} \backslash \overline{B(0, R)}$. Thus, by convexity

$$
u_{0}\left(\frac{y}{1-\lambda}\right) \geqslant u_{0}(x)+\left\langle p, \frac{y}{1-\lambda}-x\right\rangle
$$


which implies that

$$
u_{\varepsilon, \lambda}(y) \geqslant(1-\lambda)\left[u_{0}(x)-\langle p, x\rangle+\varepsilon\right]+\langle p, y\rangle .
$$

Let us define

$$
\forall z \in \mathbb{R}^{N}, \quad \pi(z)=(1-\lambda)\left[u_{0}(x)-\langle p, x\rangle+\varepsilon\right]+\langle p, z\rangle .
$$

Let us notice that, on the one hand, $\pi(x) \geqslant u_{0}(x)$ because of $[19)$, and on the other hand, $u_{\varepsilon, \lambda}(y) \geqslant$ $\pi(y)$. Therefore

$$
\left|\left(x, u_{0}(x)\right)-\left(y, u_{\varepsilon, \lambda}(y)\right)\right| \geqslant \mathrm{d}\left(\left(x, u_{0}(x)\right), \operatorname{Graph}(\pi)\right)=\gamma\left[1+|p|^{2}\right]^{-1 / 2},
$$

where $\gamma=\lambda\left(\langle p, x\rangle-u_{0}(x)\right)+(1-\lambda) \varepsilon$. Therefore, using (19), we get

$$
\left|\left(x, u_{0}(x)\right)-\left(y, u_{\varepsilon, \lambda}(y)\right)\right| \geqslant \lambda \frac{1+|p|}{\left(1+|p|^{2}\right)^{1 / 2}} \geqslant \frac{\lambda}{2} .
$$

In conclusion, we have proved that, for any $\varepsilon>0$ and any $\lambda \in\left(0, \lambda_{\varepsilon}\right)$,

$$
\mathrm{d}\left(\operatorname{Graph}\left(u_{0}\right), \operatorname{Graph}\left(u_{\varepsilon, \lambda}\right)\right) \geqslant \min \left\{\gamma_{\varepsilon} / 2, \lambda / 2\right\}>0,
$$

which completes the proof of Theorem 4.2

REMARK 4.2 Lemma 4.3 has the following geometric interpretation: Let $\mathcal{C}$ be any open convex subset of $\mathbb{R}^{N} \backslash B(0, R+1)$. Let $u_{0}^{\mathcal{C}}$ be the smallest convex function which coincides with $u_{0}$ on $\mathcal{C}$, namely

$$
\forall x \in \mathbb{R}^{N}, \quad u_{0}^{\mathcal{C}}(x)=\sup \left\{u_{0}(z)+\langle p, x-z\rangle: z \in \mathcal{C} \text { and } p \in \partial u_{0}(z)\right\} .
$$

Then inequality $(18)$ states that $u_{0}^{\mathcal{C}}$ is bounded from above by the constant $c$ on the ball $B(0,1)$.

Proof of Lemma 4.3. Let $R_{0}>0$ be some constant such that $u_{0}$ is convex on any convex subset of $\mathbb{R}^{N} \backslash B\left(0, R_{0}\right)$. Let us fix $z \in \mathbb{R}^{N}$ with $|z| \leqslant 1$ and set $u_{z}(\cdot)=u_{0}(\cdot+z)$. Then $u_{z}$ is convex on any convex subset of $\mathbb{R}^{N} \backslash B\left(0, R_{0}+1\right)$.

We claim that, for any $x \in \mathbb{R}^{N}$ with $|x|>R_{0}+2$, and any $p \in \partial u_{z}(x)$ and $q \in \partial u_{z}(y)$ where $y=\left(R_{0}+2\right) x /|x|$, we have

$$
u_{z}(x)-\langle p, x\rangle \leqslant u_{z}(y)-\langle q, y\rangle .
$$

Indeed, since the segment $[x, y]$ has an empty intersection with $B\left(0, R_{0}+1\right)$, and since $u_{z}$ is convex on any convex subset of $\mathbb{R}^{N} \backslash B\left(0, R_{0}+1\right)$, we have

$$
u_{z}(y) \geqslant u_{z}(x)+\langle p, y-x\rangle .
$$

Moreover, from the convexity of $u_{z}$ on some convex neighbourhood of the segment $[x, y]$, we have $\langle p-q, x-y\rangle \geqslant 0$. Since $x-y=\left(|x| /\left(R_{0}+2\right)-1\right) y$ with $|x|>R_{0}+2$, this implies that $\langle p, y\rangle \geqslant\langle q, y\rangle$. From this inequality and from $(21)$ we deduce that

$$
u_{z}(y) \geqslant u_{z}(x)-\langle p, x\rangle+\langle q, y\rangle
$$

which proves our claim.

Since $u_{0}$ is convex on any convex subset of $\mathbb{R}^{N} \backslash B\left(0, R_{0}\right)$, it is locally Lipschitz continuous on this set. Let $L$ be some Lipschitz constant of $u_{0}$ on $B\left(0, R_{0}+3\right) \backslash B\left(0, R_{0}+1\right)$. In particular, for any $y \in B\left(0, R_{0}+3\right) \backslash B\left(0, R_{0}+1\right)$ and any $q \in \partial u_{0}(y)$, we have $|q| \leqslant L$. 
Let us fix $z \in B(0,1)$ and $x \in \mathbb{R}^{N}$ with $|x| \geqslant R_{0}+3$. We apply (20) to $z$ and $x-z$ to obtain

$$
\forall p \in \partial u_{0}(x), \quad u_{0}(x)-\langle p, x-z\rangle \leqslant u_{0}(y+z)-\langle q, y\rangle
$$

where $y=\left(R_{0}+2\right)(x-z) /|x-z|$ and $q \in \partial u_{0}(y+z)$. Let us notice that $|q| \leqslant L$ since $y+z \in B\left(0, R_{0}+3\right) \backslash B\left(0, R_{0}+1\right)$. Therefore

$$
\forall p \in \partial u_{0}(x), \quad u_{0}(x)-\langle p, x-z\rangle \leqslant\left\|u_{0}\right\|_{L^{\infty}\left(B\left(0, R_{0}+3\right)\right)}+L\left(R_{0}+2\right) .
$$

Since this inequality holds true for any $z$ with $|z| \leqslant 1$, we finally deduce that

$$
\forall p \in \partial u_{0}(x), \quad u_{0}(x)-\langle p, x\rangle \leqslant c-|p|,
$$

with $c=\left\|u_{0}\right\|_{L^{\infty}\left(B\left(0, R_{0}+3\right)\right)}+L\left(R_{0}+2\right)$. This is the desired result if we set $R=R_{0}+3$.

\section{REFERENCES}

1. BARLES, G. Remark on a flame propagation model. Rapport INRIA, 464, 1985.

2. Barles, G., Biton, S., Bourgoing, M., \& Ley, O. Uniqueness results for quasilinear parabolic equations through viscosity solutions' methods. Calc. Var. Partial Differential Equations 18 (2003), 159179. Zbl 1036.35001 MR 2010963

3. Barles, G., Biton, S., \& LEy, O. A geometrical approach to the study of unbounded solutions of quasilinear parabolic equations. Arch. Ration. Mech. Anal. 162 (2002), 287-325. Zbl 1052.35084 MR 1904498

4. Barles, G., Biton, S., \& LEY, O. Uniqueness for parabolic equations without growth condition and applications to the mean curvature flow in $\mathbb{R}^{2}$. J. Differential Equations 187 (2003), 456-472. Zbl 1028.35067 MR 1949450

5. Barles, G., Soner, H. M., \& Souganidis, P. E. Front propagation and phase field theory. SiAM J. Control Optim. 31 (1993), 439-469. Zbl 0785.35049 MR 1205984

6. Barles, G., \& Souganidis, P. E. A new approach to front propagation problems: theory and applications. Arch. Ration. Mech. Anal. 141 (1998), 237-296. Zbl 0904.35034 MR 1617291

7. Biton, S., Chasseigne, E., \& Ley, O. Uniqueness without growth condition for the mean curvature equation with radial initial data. Comm. Partial Differential Equations 28 (2003), 1503-1526. Zbl 1030.35098 MR 2001171

8. Chen, Y. G., Giga, Y., \& Goto, S. Uniqueness and existence of viscosity solutions of generalized mean curvature flow equations. J. Differential Geom. 33 (1991), 749-786. Zbl 0696.35087 MR 1100211

9. ChOU, K.-S., \& Kwong, Y.-C. On quasilinear parabolic equations which admit global solutions for initial data with unrestricted growth. Calc. Var. Partial Differential Equations 12 (2001), 281-315. Zbl 0980.35061 MR 1825875

10. CRAndall, M. G., Ishit, H., \& LiOns, P.-L. User's guide to viscosity solutions of second order partial differential equations. Bull. Amer. Math. Soc. (N.S.) 27 (1992), 1-67. Zbl 0755.35015 MR 1118699

11. ECKer, K., \& Huisken, G. Interior estimates for hypersurfaces moving by mean curvature. Invent. Math. 105 (1991), 547-569. Zbl 0707.53008 MR 1117150

12. Evans, L. C., \& Spruck, J. Motion of level sets by mean curvature. I. J. Differential Geom. 33 (1991), 635-681. Zbl 0726.53029 MR 1100206

13. Giga, Y. Surface Evolution Equations. Monogr. Math. 99, Birkhäuser, Basel (2006). Zbl 1096.53039 MR 2238463

14. Giga, Y., Goto, S., IshiI, H., \& SAto, M.-H. Comparison principle and convexity preserving properties for singular degenerate parabolic equations on unbounded domains. Indiana Univ. Math. J. 40 (1991), 443-470. Zbl 0836.35009 MR 1119185 
15. Giga, Y., \& SAto, M.-H. On semicontinuous solutions for general Hamilton-Jacobi equations. Proc. Japan Acad. Ser. A Math. Sci. 75 (1999), 159-162. Zbl 0943.35010 MR 1740813

16. Ilmanen, T. Generalized flow of sets by mean curvature on a manifold. Indiana Univ. Math. J. 41 (1992), 671-705. Zbl 0759.53035 MR 1189906

17. IsHII, H. Degenerate parabolic PDEs with discontinuities and generalized evolutions of surfaces. $A d v$. Differential Equations 1 (1996), 51-72. Zbl 0841.35057 MR 1357954

18. Ishit, H., \& Mikami, T. Motion of a graph by R-curvature. Arch. Ration. Mech. Anal. 171 (2004), 1-23. Zbl 1053.60103 MR 2029528

19. IshiI, H., \& Souganidis, P. E. Generalized motion of non compact hypersurfaces with velocity having arbitrary growth on the curvature tensor. Tôhoku Math. J. 47 (1995), 227-250. Zbl 0837.35066 MR 1329522

20. Osher, S., \& SethiAn, J. Fronts propagating with curvature dependent speed: algorithms based on Hamilton-Jacobi formulations. J. Comput. Phys. 79 (1988), 12-49. Zbl 0659.65132 MR 0965860

21. Soner, H. M. Motion of a set by the curvature of its boundary. J. Differential Equations 101 (1993), 313-372. Zbl 0769.35070 MR 1204331

22. Soner, H. M., \& Souganidis, P. E. Singularities and uniqueness of cylindrically symmetric surfaces moving by mean curvature. Comm. Partial Differential Equations 18 (1993), 859-894. Zbl 0804.53006 MR 1218522

23. Souganidis, P. E. Front propagation: theory and applications. Viscosity Solutions and Applications (Montecatini Terme, 1995), Lecture Notes in Math. 1660, Springer, Berlin (1997), 186-242. Zbl 0882.35016 MR 1462703 